\title{
Correction: Melatonin suppresses chronic restraint stress-mediated metastasis of epithelial ovarian cancer via NE/AKT/ $\beta$-catenin/SLUG axis
}

Shixia Bu, Qian Wang $\mathbb{D}$, Junyan Sun, Xiao Li, Tingting Gu and Dongmei Lai

Correction to: Cell Death and Disease https://doi.org/10.1038/s41419-020-02906-y published online 18 August 2020

The original version of this article contained errors in the author affiliations.

Author Xiao Li was incorrectly associated with Department of Obstetrics and Gynaecology, Shanghai Sixth People's Hospital, Shanghai Jiaotong University,
Shanghai 200233, China. The correct affiliation is Shanghai Municipal Key Clinical Speciality, Shanghai 20030, China.

This has now been corrected in both the PDF and HTML versions of the article.

Published online: 08 September 2020 